\title{
Propriedades funcionais do yacon e atomização por diferentes agentes encapsulantes
}

\author{
Functional properties of yacon and atomization by different encapsulating agents \\ Propiedades funcionales del yacón y atomización por diferentes agentes encapsulantes
}

Recebido: 09/09/2021 | Revisado: 15/09/2021 | Aceito: 20/09/2021 | Publicado: 21/09/2021

\author{
Amanda Fernandes Oliveira de Souza \\ ORCID: https://orcid.org/0000-0002-2284-0508 \\ Universidade Federal Rural de Pernambuco, Brasil \\ E-mail: Amandafernandes.afos@gmail.com \\ Eduardo do Nascimento Filho \\ ORCID: https://orcid.org/0000-0001-7932-9308 \\ Universidade Federal Rural de Pernambuco, Brasil \\ E-mail: edunascimento50@gmail.com \\ Thaynna Leocádio Trajano Lacerda Sousa \\ ORCID: https://orcid.org/0000-0002-2670-8950 \\ Universidade Federal Rural de Pernambuco, Brasil \\ E-mail: thaynna.leocadio0@gmail.com \\ Celiane Gomes Maia da Silva \\ ORCID: https://orcid.org/0000-0003-0700-5499 \\ Universidade Federal Rural de Pernembuco, Brasil \\ E-mail: celiane.msilva@ufrpe.br
}

\begin{abstract}
Resumo
A batata yacon é uma raiz tuberosa que vem despertando interesse, por apresentar em sua composição compostos bioativos que oferecem benefícios à saúde. Apesar de ser bastante perecível, apresenta grande potencial na tecnologia no desenvolvimento de novos produtos alimentícios. $\mathrm{O}$ artigo tem por objetivo promover informações acerca do yacon que é considerado alimento funcional, uma vez que apresenta um alto teor de compostos fenólicos, apresentando características prebióticas e antioxidantes, respectivamente, e sua aplicação da tecnologia de alimentos com o emprego do processo de atomização. O processo de microencapsulação apresenta vantagens visando a preservação das características originais de produtos naturais. O yacon em pó a partir da atomização do extrato, com a utilização de agentes encapsulantes como maltodextrina e goma arábica é uma alternativa viável para a preservação de alimentos com alto valor agregado, além de contribuir para a manutenção da estabilidade e dos compostos fenólicos, mesmo em altas temperaturas. Sendo assim, o desenvolvimento desse produto caracteriza-se como uma inovação importante para a indústria de alimentos funcionais.
\end{abstract}

Palavras-chave: Alimentos funcionais; Prebióticos; Microencapsulação de Células.

\begin{abstract}
The yacon potato is a tuberous root that has been arousing interest, as its composition contains bioactive compounds that offer health benefits. Despite being quite perishable, it has great potential in technology for the development of new food products. The article aims to provide information about yacon, which is considered a functional food, since it has a high content of phenolic compounds, prebiotic and antioxidant characteristics, respectively, and its application of food technology using the atomization process. The microencapsulation process is characterized by the preservation of the original attributes of natural products. Yacon powder from the atomization of the extract, with the use of encapsulating agents such as maltodextrin and gum arabic, is a viable alternative for the preservation of foods with high added value, in addition to contributing to the maintenance of stability and phenolic compounds, even at high temperatures. Therefore, the development of this product represents an important innovation for the food industry.
\end{abstract}

Keywords: Functional Food; Prebiotic; Cell Microencapsulation.

\section{Resumen}

Yacón es una raíz tuberosa que ha despertado interés, ya que su composición contiene compuestos bioactivos que ofrecen beneficios para la salud. A pesar de ser bastante perecedero, tiene un gran potencial en tecnología para el desarrollo de nuevos productos alimenticios. El artículo tiene como objetivo brindar información sobre el yacón, que se considera un alimento funcional, ya que tiene un alto contenido en compuestos fenólicos, características prebióticas y antioxidantes, respectivamente, y su aplicación de tecnología alimentaria mediante el proceso de atomización. El proceso de microencapsulación se caracteriza por la preservación de los atributos originales de los productos naturales. El polvo de yacón procedente de la atomización del extracto, con el uso de agentes encapsulantes como 
maltodextrina y goma arábiga, es una alternativa viable para la conservación de alimentos con alto valor agregado, además de contribuir al mantenimiento de la estabilidad y compuestos fenólicos, incluso a altas temperaturas. Por lo tanto, el desarrollo de este producto representa una innovación importante para la industria de alimentos.

Palabras clave: Alimentos Funcionales; Prebiótico; Microencapsulación celular.

\section{Introdução}

A obesidade se tornou uma epidemia em todo o mundo e está associada a uma morbidade e mortalidade significativa. A prevalência mundial da obesidade quase triplicou entre 1975 e 2016. De acordo com estimativas da Organização Mundial da Saúde (OMS) no ano de 2016 cerca de 2 bilhões de adultos (39\%) estavam com sobrepeso e mais de 650 milhões obesos (WHO, 2019). A preocupação com o aumento da prevalência de excesso de peso deve-se principalmente as consequências para a saúde, pois representa um importante fator de risco para as doenças crônicas não transmissíveis (WHO, 2016; Lavie et al., 2018).

Alimentos funcionais podem regular o sistema imunológico por meio do aumento da resposta imunológica, que fornece as defesas do hospedeiro contra infecções e inflamação (Hachimura et al., 2018). A produção desses componentes alimentares, como prebióticos, vem crescendo com o intuito de auxiliar no combate a problemas crônicos de saúde como a doença hepática gordurosa não alcoólica (DHGNA), câncer, obesidade, diabetes tipo II e doenças cardiovasculares. As definições atribuídas aos alimentos funcionais são muitas e incluem alimentos comercializados que beneficiam à saúde, fontes de alimentos com propriedades fisiológicas favoráveis além de seus usos nutricionais de fornecimento de nutrientes essenciais e substâncias naturais que podem ser consumidas diariamente destinadas à regular e melhorar um sistema imunológico, além do seu papel nutricional (Rivera \& Bocanegra-García, 2010; Maroyi, 2019; Silva, et al., 2020).

A raiz tuberosa de yacon (Smallanthus sonchifolius) é considerada uma fonte natural de frutooligossacarídeos (FOS), inulina e proporciona uma fermentação seletiva no cólon pelas Bifidobacterium spp. e Lactobacillus spp (Mejía-Aguila, et al., 2020). Por isso, é adicionado a muitos produtos para promover a manutenção da microbiota intestinal saudável (Sousa et al., 2015; Oliveira et al., 2016; Brandão et al., 2019). As raízes do yacon são passíveis às reações de escurecimento enzimático e apresentam elevado teor de água (70\% a $90 \%$ do peso fresco). É muito perecível, deteriora-se rapidamente devido ao seu alto teor de umidade e atividade enzimática. Com isso, eleva-se o índice de perdas durante a pós-colheita, consequentemente, há perdas econômicas, restringindo o desenvolvimento sustentável da cultura (Shi et al., 2013).

A microencapsulação por spray dryer a partir de extrato de yacon vem sendo utilizada na tecnologia de alimentos visando à proteção de compostos instáveis presentes naturalmente no yacon e têm demonstrado resultados relativamente satisfatórios nas análises físico-químicas como as de FRAP (Ferric Reducing Antioxidant Power) e DPPH (2,2-difenil-1picrilidrazil), que a quantificam os antioxidantes por exemplo. Durante o processo de microencapsulação, uma substância (agente ativo), no estado sólido, líquido ou gasoso, é aprisionada dentro de pequenas cápsulas, de tamanho variado (Krishna \& Jyothika, 2015).

Essa técnica protege moléculas sensíveis à luz, calor, umidade ou oxidação (Aizpurua-Olaizola et al., 2016), além de melhorar a biodisponibilidade e estabilidade das moléculas e dos produtos, torna-se bastante interessante para aplicação em extratos contendo compostos fenólicos e outros compostos bioativos (Aguiar et al., 2017).

Agentes encapsulantes ou material de parede têm um papel crucial no processo de encapsulação, sua natureza é considerada um dos principais fatores que influenciam a estabilidade (Özkan \& Bilek, 2014). A escolha do material de parede apropriado é a etapa principal no encapsulamento de secagem por spray dryer. Algumas das propriedades ideais incluem: baixa viscosidade, baixa higroscopia, capacidade de estabilizar o material do núcleo em emulsão, alta solubilidade, capacidade de formação de filme, fornece alta proteção, ausência de sabor e baixo custo (Franco et al., 2017). Sabendo que a raiz de yacon é fonte de fibras prebióticas que contribuem para o equilíbrio da microflora intestinal, mantendo a saúde do indivíduo, o 
objetivo deste artigo é apresentar a importância das propriedades funcionais presentes no yacon voltada para a aplicação da tecnologia de alimentos e o emprego do processo de atomização.

\section{Metodologia}

Para elaboração do presente artigo, foi realizado um estudo de revisão de literatura científica de artigos originais, que tratavam das características funcionais do yacon, qualidade nutricional e atomização com diferentes agentes encapsulantes. Foram utilizados publicações de revistas disponíveis nas bases de dados Google Acadêmico, SciELO, Scopus e Science Direct, nos idiomas português, inglês e espanhol, dentro da serie temporal de 1991 à 2021, com os termos yacon, prebiótico e atomização, onde o critério de inclusão foram os artigos com maior relevância para ciências agrárias e como critério de exclusão artigos que utilizassem outros tipos de matérias de parede que não fossem maltodextrina e goma arábica. Em seguida, os estudos encontrados foram analisados quanto aos seus respectivos títulos e resumos referente à pertinência aos temas de enfoque do presente artigo.

\section{Resultados e Discussão}

\subsection{Características da batata Yacon}

Yacon (Smallanthus sonchifolius) (Figura 1) é uma raiz que pertence à família botânica Asteraceae (Caetano et al., 2016) e uma rica fonte de FOS e compostos fenólicos, particularmente ácido clorogênico (Silva et al., 2018). O nome yacon é composto de duas palavras yakku = insípido e unu = água, significando água sem sabor (Zardini, 1991). Apresenta características vantajosas sobre as demais raízes tuberosas pela sua alta produtividade, controle da erosão, adaptabilidade às variedades de climas e solos. E também possui qualidades na alimentação, como por exemplo, proporciona uma alimentação de baixa caloria ( $\pm 33 \mathrm{Kcal}$ em $100 \mathrm{~g}$ de yacon cru) e com propriedades medicinais auxiliando na redução da glicose no sangue e estimulando o crescimento das bactérias benéficas do intestino (Grau \& Rea, 1997).

No Brasil as raízes de yacon são geralmente consumidas como frutas, pois são suculentas e doces como uma maçã (De Andrade et al., 2014). Originalmente o yacon era cultivado na região andina na América do Sul, o Peru é o país onde há maior biodiversidade dessa raiz tuberosa. Devido as suas características, houve uma expansão para outras regiões fora dos Andes. Ela também é cultivada no Brasil, República Tcheca, China, Japão, Nova Zelândia, Filipinas, Rússia, Coréia do Sul e EUA (Honor'e, 2015).

Figura 1. Batata Yacon.

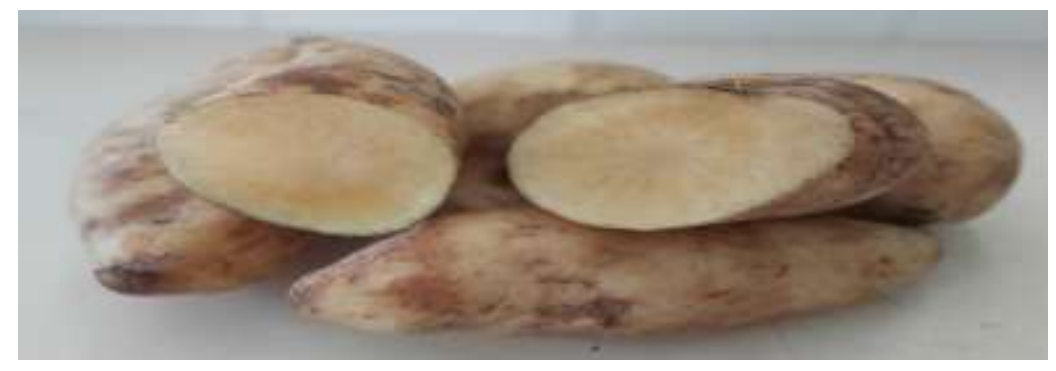

Fonte: Autores (2020).

Em contraste com a maioria das raízes comestíveis de armazenamento de amido, o yacon contém grandes quantidades de FOS, que constituem um monômero de glicose $(G)$ ligado por $\beta(2 \rightarrow 1)$ à unidades de frutose $(F)$, formando uma mistura de 1-kestose (GF2), nistose (GF3) e 1Fß-frutofuranosil nistose (GF4), juntamente com outros GFs (Silva et al., 2018). 
Além disso, métodos analíticos evidenciam seu potencial medicinal que está relacionado ao seu alto teor de compostos bioativos (Russo et al., 2015) como os flavonóides, compostos fenólicos e triptofano com atividade antioxidante, antiinflamatória e propriedade anticancerígena (Delgado et al., 2013). A raiz apresenta baixo teor calórico com mais de 70\% de água e é rica em fibra (aproximadamente $45 \mathrm{~g}$ em 100g de matéria seca), principalmente e FOS (Caetano et al., 2016).

O FOS e inulina são conhecidos como fontes de prebióticos com efeito benéfico à saúde humana (Fernandez et al., 2013; Dwivedi et al., 2014). Assim, a principal funcionalidade apresentada pelo yacon é o seu potencial prebiótico, pois nele há compostos que são facilmente metabolizados e podem estimular o crescimento de bactérias benéficas (Souza et al., 2018, Reis et al., 2021).

Campos et al. (2012) obtiveram resultados positivos, ao testar o efeito prebiótico do yacon in vivo. Concluíram que uma dieta rica em FOS promoveu o crescimento das bactérias benéficas como as Bifidobacterium spp. e Lactobacillus spp, resultando em níveis elevados de Ácidos Graxos de Cadeia Curta (AGCC). Assim, os resultados mostrados evidenciaram benefícios para a saúde do cólon intestinal. No entanto, o yacon é um alimento altamente perecível, mesmo quando armazenado sob refrigeração (Shi et al., 2013). A desidratação de raízes de yacon sem adição de conservantes ou químicos é um processo simples para garantir um produto natural com grande estabilidade, principalmente de FOS (Caetano et al., 2016).

\subsection{Propriedades Funcionais do Yacon}

É crescente a procura por alimentos funcionais, como também o incentivo para inovação na elaboração de novos produtos alimentícios (Kumar et al., 2015; Parussolo et al., 2017). Os alimentos considerados funcionais são definidos como produtos alimentares convencionais, que faz parte da dieta normal e demonstram benefícios para a saúde, além de suas propriedades nutricionais (Rai et al., 2016). Os componentes que são considerados funcionais muito estudados são os antioxidantes, ácidos graxos insaturados, prebióticos e probióticos (Al-Sheraji et al., 2013; Yasmin et al., 2015). As raízes de yacon têm sido estudadas como alimentos funcionais, devido a sua alta concentração de FOS e componentes antioxidantes (Jimenéz \& Sammán, 2014; Sousa et al., 2015). O FOS é um prebiótico que garante propriedades promotoras de saúde (Caetano et al., 2016).

O estudo randomizado, duplo cego, realizado por Machado et al. (2018) avaliou o consumo diário de $25 \mathrm{~g}$ da farinha de yacon $(0,1 \mathrm{~g}$ de FOS $/ \mathrm{kg}$ de peso corporal / dia) durante 6 semanas em adultos com excesso de peso. Os resultados demonstram que a ingestão dessa farinha apresenta uma quantidade adequada de fibras que melhoram a consistência das fezes e há uma diminuição na constipação intestinal. O consumo de yacon provoca uma diminuição do peso e gordura corporal e circunferência da cintura além disso, leva a redução de glicose através de seus efeitos inibitórios sobre a $\alpha$-glucosidase, enzima responsável pela absorção intestinal de glicose. Com essa inibição, ocorre uma redução na absorção de glicose pelo intestino, reduzindo as concentrações de glicose pós-prandial no sangue (Zhen-yuan et al., 2014).

O yacon também tem outro constituinte que pode ter efeitos hipoglicêmicos, o ácido clorogênico (ácido 3-O-cafeoilD-quínico (CGA), um éster formado por ácidos caféico e químico, que é um dos principais compostos polifenólicos do yacon. O CGA pode modular a concentração plasmática de insulina e inibir a gliconeogênese hepática (Genta et al., 2009; Ojansivu et al., 2011), devido à inibição da glicose6-fosfatase, enzima que catalisa a etapa final de glicogenólise e gliconeogênese (Arion et al., 1998; Herling et al., 1998).

Em um estudo experimental realizado por Dionisio et al. (2015), foi testada uma bebida funcional com yacon e suco de caju. Foram divididos em três grupos: grupo controle, diabéticos não tratados e diabéticos em tratamento com 100, 200 ou $400 \mathrm{mg}$ de bebida funcional liofilizada, administradas em 30 dias. Os resultados mostraram que a bebida funcional promoveu o crescimento da atividade de lactobacilos da microflora fecal de ratos wistar em todas as concentrações testadas. Sivieri et al. (2014) investigaram a ação prebiótica de um extrato aquoso de yacon (AYE) no Simulador de Ecossistema Microbiano 
Intestinal Humano (SHIME), e observaram um aumento seletivo de bactérias probióticas nas populações de Bifidobacterium spp. e Lactobacillus spp., e na produção de ácidos graxos de cadeia curta no sistema SHIME. Esses resultados foram positivos em relação ao potencial como propriedades funcionais presentes no yacon. Assim, a microencapsulação por atomização é um processo eficaz para aumentar o tempo de vida útil a partir do extrato de yacon, que além de preservar a qualidade nutricional, agrega valor ao alimento.

\subsection{Microencapsulação e secagem por atomização (Spray dryer)}

A tecnologia de microencapsulação é uma ferramenta baseada na formação de uma estrutura de proteção, abrange uma substância de interesse para proteção e liberação controlada (Alvim et al., 2016). Teve início no ano de 1950, com a patente de Green e Schleicher relativa à produção de cápsulas (Ghosh, 2006). Essa tecnologia tem como base uma criação de uma barreira (matriz) entre a substância de interesse (substância ativa) e o ambiente (alimentos) (Gharsallaoui et al., 2007).

$\mathrm{Na}$ área de alimentos, a técnica é empregada visando à proteção de compostos instáveis presentes em diversos produtos. Essa tecnologia propicia, assim, uma barreira entre o composto ativo e o meio, promovendo a estabilidade da substância bioativa, uma vez que reduz o processo de degradação, mantendo sua propriedade funcional, bem como aumentando a sua biodisponibilidade (Fang \& Bhandari, 2010; Paulo \& Santos, 2017).

A microencapsulação é um processo que permite a proteção de substâncias encapsuladas sensíveis, contra as condições de agentes externos, como a presença de luz, calor, umidade, radiação e oxigênio (Gonçalves, Estevinho \& Rocha, 2016), além de permitir a liberação controlada dessas substâncias aprisionadas (Bansode et al., 2010; Byun et al., 2010). As microcápsulas possuem diferentes morfologias, dependendo da técnica empregada no processo de microencapsulação, bem como, do tipo de material de parede e do núcleo (Fang \& Bhandari, 2010; Vila et al., 2015).

O material da parede deve possuir alta estabilidade, emulsificação e proteção, evitando assim a separação do material do núcleo durante secagem por pulverização. Geralmente, os materiais de parede usados para microencapsulação são gomas, polissacarídeos, proteínas e suas misturas (Bora et al., 2018). Dentre as características a serem consideradas na escolha do material de parede está o custo, a capacidade de formar filmes, a higroscopicidade, o teor de sólidos, a viscosidade e a biodegradabilidade (Madene et al., 2006; Bora et al., 2018).

A secagem por atomização é uma operação comum da unidade para converter materiais líquidos em pó, inventado por Samuel R. Percy e tem sido usado por quase 150 anos desde 1872 (Cao et al., 2000). Em alimentos, a atomização é um dos métodos comuns usados devido ao processo rápido e econômico. Apresenta baixo teor de umidade, atividade da água (Aw), higroscopicidade e estabilidade dos compostos bioativos (Tontul \& Topuz, 2017). Assim, o pó constituído possui propriedades desejáveis para facilitar o transporte, armazenamento e aplicação em alimentos (Desai \& Hyn-Jin, 2005).

As partículas são facilmente reconstituídas, sendo bastante interessante para aplicações em alimentos líquidos e pastosos, bem como em pós-instantâneos (Souza et al., 2018). O processo de secagem por atomização consiste em três etapas sequenciais: a atomização da amostra do alimento em suspensão, a secagem de gotículas líquidas e a recuperação do pó (Shishir \& Chen, 2017). Fatores como o material de parede, condições de secagem e temperaturas de entrada e saída, vazão, tipo de atomizador, todos podem afetar a microestrutura das cápsulas produzidas (Oliveira \& Petrovick, 2010; Shishir \& Chen, 2017).

\subsection{Agente Encapsulante}

Os agentes encapsulantes são formadores de filmes protetores, tem como principais características desejáveis propiciar baixa higroscopicidade, baixa viscosidade, baixa umidade, capacidade de emulsificar e estabilizar o material do núcleo, baixa reatividade, máxima proteção contra luz, pH e oxigênio, ausência de sabor e odor desagradáveis e baixo custo 
(Cano-higuita et al., 2015). Devem ser considerados também a toxicidade, eficiência do encapsulamento, estabilidade, grau de proteção do núcleo e propriedades microscópicas da superfície (Bora et al., 2018).

$\mathrm{O}$ agente encapsulante à base de hidrocolóides como maltodextrina e goma arábica é usualmente utilizado. O estudo realizado Kingwatee et al. (2015) utilizou a maltodextrina e goma arábica para microencapsulação por atomização de sucos de lichia de secagem por spray com Lactobacillus casei 01 , obtiveram resultados satisfatórios na redução da viscosidade, teor de umidade, Aw e higroscopicidade do microencapsulado.

A Goma Arábica é um composto natural de proteínas e polissacarídeos, é muito utilizado como material de secagem por pulverização. Sua estrutura contém um grande grupo com alta ramificação de polissacarídeo (97\%) constituído por uma espinha dorsal de unidades de galactose com ramos ligados de arabinose e ramnose. O segundo grupo é um complexo de arabinogalactano-proteína $(<3 \%)$ de maior peso molecular (Montenegro et al., 2012; Arslan-tontul \& Erbas, 2017). É considerada como um dos agentes encapsulantes mais importantes, pois é um polímero natural de baixa viscosidade, solubilidade adequada, boa capacidade emulsificante em soluções aquosas, é atóxica, inodora, insípida e tem sido utilizada no encapsulamento de alimentos por muitos anos, no entanto tem baixa disponibilidade e elevado custo, quando comparado a outros agentes encapsulantes (Estevinho et al., 2013; Chranioti \& Tzia, 2014;).

Um estudo realizado por Rezende, Nogueira, e Narain, (2018), ao analisarem a polpa de acerola e os extratos de seus resíduos, após a microencapsulação por spray, utilizando goma arábica e maltodextrina como agentes encapsulantes a uma temperatura de $170{ }^{\circ} \mathrm{C}$ observaram que o resultado de FRAP e ORAC (Capacidade de Absorção de Radicais de Oxigênio) apresentaram maior atividade antioxidante, em comparação com outros métodos analisados como o DPPH e ABTS (2,2-azinobisethylbenzo-thiazoline6-sulfonic acid). Resultado semelhante foi encontrado no estudo de Do Carmo et al. (2017) obtendo elevada estabilidade e atividade antioxidante do extrato em pó de beterraba após a atomização por spray dryer usando maltodextrina, inulina e isolado de proteína de soro de leite como agentes encapsulantes.

As maltodextrinas são amidos hidrolisados do trigo, batata e milho, com diferentes graus de equivalência de dextrose de 10 a 20, esses polissacarídeos de alto peso molecular não tem sabor aparente, é facilmente biodegradável e fornece um bom encapsulamento com baixo custo (Özkan \& Bilek, 2014). As maltodextrinas são carboidratos que podem contribuir para aumentar a estabilidade das bactérias secas por pulverização em termos de Aw, teor de umidade, pH, solubilidade, higroscopicidade, composição nutricional, temperatura de transição vítrea, cor e fluidez (Sosa, et al., 2016). São materiais solúveis em água, protege o ingrediente encapsulado da oxidação, têm baixa viscosidade, elevado teor de sólidos e estão disponíveis em diferentes pesos moleculares, o que fornece diferentes densidades de material de parede ao redor dos materiais de recheio (agentes encapsulados) que são sensíveis a oxidação e degradação (Bora et al., 2018).

A eficácia da maltodextrina como agente encapsulante de extratos vegetais contendo compostos bioativos, como compostos fenólicos é demonstrada em diversos estudos com diferentes composições de matrizes alimentares (Santos et al., 2017; Nunes et al., 2018). Um estudo realizado por Tengse et al. (2017) utilizaram maltodextrina como agente encapsulante no processo de atomização de extrato de chá verde, como resultado obtiveram as microcápsulas com alto teor de polifenóis e elevada atividade antioxidante, 72,91\% de polifenóis livres e 57,81mg/g de matéria, expressa como equivalente de ácido gálico, com 0,92 de conveniência.um pesquisa realizada por Santos et al. (2017), ao microencapsular o suco de amora-preta (Rubus fruticosus) com a maltodextrina observou a eficácia na proteção dos compostos fenólicos e das antocianinas, durante o tempo de armazenamento estudado de 36 dias. Já em matrizes amiláceas a maltodextrina quando em conjunto com a goma arábica contribui para estabilização das microcápsulas assim aumentando seu rendimento, isso pode ser observado no estudo realizado por Dhiman et al. (2021) ao utilizarem a maltodextrina e goma arábica para o encapsulamento do suco de beterraba por atomização, no qual a eficiência de encapsulação foi de $73,27 \%$, apresentando um teor de umidade muito baixo $(1,26 \%-$ $2,88 \%$ ), o que torna o produto microbiologicamente seguro. 


\section{Conclusão}

As pesquisas relacionadas à área de alimentos utilizando yacon e seus derivados se destacam pelo potencial prebiótico, atividade antioxidante e propriedades funcionais que contribuem para melhora do sistema imune e redução da glicemia. Em função dos benefícios nutricionais a atomização a partir do extrato de yacon é uma técnica promissora para obtenção do pó com potencial aplicação em alimentos, visto que suas propriedades funcionais estão sendo cada vez mais pesquisadas e comprovadas cientificamente.

\section{Referências}

Aguiar, J., Costa, R., Rocha, F., Estevinho, B. N., \& Santos, L. (2017) Design of microparticles containing natural antioxidants: Preparation, characterization and controlled release studies. Powder Technology, 313, 287-292.

Aizpurua-olaizola, O., Navarro, P., Vallejo, A., Olivares, M., Etxebarria, N., \& Usobiaga, A. (2016). Microencapsulation and storage stability of polyphenols from Vitisvinifera grape wastes. Food Chemistry, 190, 614-621.

Al-sheraji, S. H., Ismail, A., Manap, M. Y., Mustafa, S., Yusof, R. M.; \& Hassan, F. A. (2013). Prebiotics as functional foods. A review.Journal of functional foods, $5,1542-1553$.

Alvim, I. D., Stein, M. A., Koury, I. P., Dantas, F. B. H, \& Cruz, C. L. D. C. V. (2016). Comparison between the spray drying and spray chilling microparticles contain ascorbic acid in a baked product application. Food Science and Technology. 65, 689-694.

Arion, W. J., Canfield, W. K., Ramos, F. C.., Su, M. L., Burger, H. J., \& Hemmerle H. (1998). Chlorogenic acid analogue S 3483: a potent competitive inhibitor of the hepatic and renal glucose-6-phosphatase systems. Archives of Biochemistry and Biophysics, 351, $279-285$.

Arslan-Tontul, S. \& Erbas, M. (2017). Single and double layered microencapsulation of probiotics by spray drying and spray chilling. LWT-Food Science and Technology, 81,160-169.

Bansode, S. S., Banarjee, S. K., Gaikwad, D. D., Jadhav, S. L., \& Thorat, R. M. (2010). Microencapsulation: a review. International Journal of Pharmaceutical Sciences Review and Research, 1(2), 38-43.

Bora, A. F. M., Ma, S., Li, X., \& Liu, L. (2018). Application of microencapsulation for the safe delivery of green tea polyphenols in food systems: Review and recent advances. Food Research International, 105, 241-249.

Brandão, C. C., Asquieri, E. R., \& Damiani, C. (2019). Bebida fermentada de yacon (Smallanthus sonchifolius): parâmetros do processo fermentativo. Brazilian Journal of Food Research, 10 (1), 32-46.

Byun, Y., Kim, Y. T., Desai, K. G. H., \& Park, H. J. (2010). Microencapsulation techniques for food flavour. In A. Herrmann (Ed.), The chemistry and biology of volatiles. 307-332.

Caetano, B. F. R., Moura,N. A., Almeida,A. P. S., Dias, M. C., Sivieri,K., \& Barbisan, L. F. (2016). Yacon (Smallanthus sonchifolius) as a food supplement: healthpromoting benefits of fructooligosaccharides. Nutrients, 8 (7), 436.

Campos, D., Betalleluz-pallardel, I., Chirinos, R., Aguilar-galvez, A., Noratto, G., \& Pedrescho. (2012). Prebiotic effects of yacon ( Smallanthus sonchifolius Poepp. \& Endl), a source of fructooligosaccharides and phenolic compounds with antioxidant activity. Food Chemistry, $135,1592-1599$.

Cano-higuita, D. M., Vélez, H. A. V., \& Telis, V. R. N. (2015). Microencapsulation of turmeric oleoresin in binary and ternary blend of gum arabic, maltodeztrin and modified starch. Ciência E Agrotecnologia, 39(2),173-182.

Cao, X. Q., Vassen, R., Schwarttz, S. W., Jungen, F., Tietz, D., \& Stover. (2000). Spray-drying of ceramics for plasma-spray coating, Journal of the European Ceramic Society. 20, 2433-2439.

Chranioti, C., \& Tzia, C. (2014). Arabic gum mixtures as encapsulating agents of freezedried fennel oleoresin products. Food Bioprocess Technology. $7(2), .1057-1065$.

De andrade, W. F., De souza, A., Leone, R., Ellendersen, L. N., \& Masson, M. L. (2014). Phenolic profileandantioxidantactivityofextractsofleavesand flowersofyacon (Smallanthus sonchifolius). Industrial Crops and Products, 62, 499-506.

Delgado-amaya, L.; Herrera-lópes, E.J.; Arrizon, J., Arellano-plaza, M., \& Gschaedler, A. (2013). Performance evaluation of Pichia kluyveri, Kluyveromyces marxianus and Saccharomyces cerevisiae in industrial tequila fermentation. World Journal Microbiology Biotechnology, 29(5), 875-81.

Desai, K.G.H., \& Hyun-jin, P., (2005). Recent developments in microencapsulation of food ingredients. Drying Technol. 23 (7), $1361-1394$.

Dhiman, A., Suhag, R., Chauhan, D. S., Thakur, D., Chhikara, S., \& Prabhakar, P. K. (2021). Status of beetroot processing and processed products: Thermal and emerging technologies intervention. Trends in Food Science \& Technology, 114, 443-458.

Dionisio,A., Silva,B., Vieira,C., Goes,C., Wuelitzer, A., Borges,A., Brito, A., Lonta, B., \& Figueiredo, C. (2015). Cashew-apple (Anacardium occidentale L.) and yacon (Smallanthus sonchifolius) functional beverage improve the diabetic state in rats. Food Research International, $14,3$. 
Do Carmo, E. L., et al (2017). Stability of spray-dried beetroot extract using oligosaccharides and whey proteins. Food Chemistry, S0308814617320472.Dwivedi, S., Sahrawat, K. N., \& Puppala, R. (2014). Ortiz,Plant prebiotic sand human health: biotechnology to breed prebiotic-rich nutrious food crops, Electronic Journal of Biotechnology, 17, 38245.

Estevinho, B. N., Rocha, F., Santos, L., \& Alves, A. (2013). Microencapsulation with chitosan by spray drying for industry applications - A review, Trends in Food Science \& Technology, 31,138-155.

Fang, Z., \& Bhandari, B. (2010). Encapsulation of polyphenols - a review. Trends in Food Science and Technology, 21(10), 510-523.

Fernández, E., Rajchl, A., Lachman, J. H., “Cízková, F., KvasniC. K. A., Kotíková, Z., Milella, L., M. (2013). Vold`rich, Impact of yacon landraces cultivated in the Czech Republicand their ploidy on the short-and long chain fructooligosaccharides content in tuberous roots. Food Scienc and Technology, 54,8086 .

Franco, D., Antequera, T., Pinho, S. C., Jiménez, E., Pérez-palacios, T., Fávaro-trindade, C. S., Lorenzo, J. M. (2017). The use of microencapsulation by spray-drying and its application in meat products. Strategies for Obtaining Healthier Foods. 333-362.

Genta, S., Cabrera, W., Habib, N.; Pons, J., Carillo, I.M.; \& Grau, A. (2009). Yacon syrup: Beneficial effects on obesity and insulin resistance in humans. Clinical Nutrition, 28, 182-187.

Gharsallaoui, A., Roudaut, G., Chambin, O., A. Voilley, A., \& Saurel, R. (2007). Applications of spray-drying in microencapsulation of food ingredients: An overview. Food Research International, 40, 1107-1121.

Ghosh, S. K. (2006). Functional Coatings and Microencapsulation: A General Perspective, first ed., Weinheim, WILEY-VCH Verlag GmbH and CO KGaA.

Gonçalves, A., Estevinho, B. N, \& Rocha, F. (2016). Microencapsulation of vitamin A: a review. Trends in Food Science \& Technology, 51, 76-87.

Grau, A.; \& Rea, J. (1997) Yacon.Smallanthus sonchifolius (Poep.\&Endl.)H.Robinson. In: Hermann, M.; Heller, J. (Eds.). Andean roots and tubers: ahipa, arracacha, maca and yacon. Promoting the conservation and use of.Underutilized and neglected crops.(Institute of Plant Genetics and Crop Plant Research).Gatersieben/International Plant Genetic Resources Institute.Rome, Italy, 199- 242.

Hachimura, S., Totsuk, M. A., \& Hosono, A. (2018). Immunomodulation by food: impact on gut immunity and immune cell function Bioscience. Biotechnology, and Biochemistry. 82 (4), 584-599.

Herling, A. W., Burger, H. J., Schwab, D., Hemmerle, H., Below, P., \& Schubert, G. (1998) Pharmacodynamic profile of a novel inhibitor of the hepatic glucose6-phosphatase system. American Journal of Physiology-Gastrointestinal and Liver Physiology, 274, 1087-1093.

Honor'e. M., Genta, S. B., \& Sanchez, S. S. (2015). "Smallanthus sonchifolius (yacon) leaves: an emerging source of compounds for diabetes management," Journal of Research in Biology, 5(5), 21-42.

Jiménez, M. E., \& Sammán, N. (2014). Caracterización química y cuantificación de fructooligosacáridos, compuestos fenólicos y actividad antirradical de tubérculos y raíces andinos cultivados en el noroeste de Argentina. Archivos Latinoamericanos de Nutrición, 64, 131-138.

Kingwatee, N.; Apichartsrangkoon, A.; Chaikham, P.; Worametrachanon, S.; Techarung, J.; Pankasemsuk, T. (2015). Spray drying Lactobacillus casei 01 in lychee juice varied carrier materials. LWT - Food Science and Technology, 62(1), 847-853.

Krishna, S. A., \& Jyothika, M. A (2015) Review on Microcapsules. Journal of Pharmaceutical Sciences, 4 (2), 26-33.

Kumar, H., Salminen, S., Verhagen, H., Rowland, I., Heimbach, J., Banares, S., Young, T., Nomoto, K., \& Lalonde, M. (2015). Novel probiotics and prebiotics: road to the market. Current Opinion in Biotechnology, 32, 99-103.

Lavie, C.J., Laddu, D., Arena, R., Ortega, F.B., Alpert, M.A., \& Kushner, R.F. (2018). Reprint of: healthy weight and obesity prevention: JACC health promotion series. Journal of the American College of Cardiology.72 (13),1506-1531.

Machado, A. M., Da silva, N. B. M., Chaves, J. B. P., \& Alfenas, R. C. G. (2018). Consumption of yacon flour improves body composition and intestinal function in overweight adults: A randomized, double-blind, placebo-controlled clinical trial. Clinical Nutrition. 29, $22-29$.

Madene, A., Jacquot, M., Scher, J., \& Desobry, S.A. (2006). Flavour encapsulation and controlled release - A review. International Journal of Food Science and Technology, 4(1), 1-21.

Montenegro, M. A., Boiero, M. L., Valle, L., \& Borsarelli C.D. (2012). Gum arabic: more than edible emulsifier. In: Verbeek, Journal Citation Reports. (Ed.), Products and Applications of Biopolymers. InTech, Rijeka, Croatia, 25.

Maroyi, A. (2019). Utilization of Bridelia mollis as herbal medicine, nutraceutical and functional food in southern Africa: a review. Tropical Journal of Pharmaceutical Research, 18 (1) 203-209.

Mejía-Aguila, R. A., Aguilar-Galvez, A., Chirinos, R., Pedreschi, R., \& Campos, D. (2020). Vacuum impregnation of apple slices with yacon (Smallanthus sonchifolius Poepp. \& Endl) fructooligosaccharides to enhance the functional properties of the fruit snack. International Journal of Food Science and Technology, 56, 392-401.

Nunes,G. L.,Etchepare,M. A.,Cichoski,A. J.,Zepka,L. Q., Lopes,E. J., \& Barin,J. S. (2018). Inulin,himaizend trehalose as thermal protectants for increasing viability of Lactobacillus acidophilus encapsulated by spray drying. Food Science and Technology, 89, 128-133.

Ojansivu, I., Ferreira, C. L., \& Salminen, S. (2011). Yacon, a new source of prebiotic oligosaccharides with a history safe of use. Trends in Food Science and Technology, 22,.40-46. 
Oliveira, L. F., Correa, J. F. G., Pereira, M. C. A., Ramos, A. L. S., \& Vilela, M. B. (2016). Dsmotic dehydration of yacon (Smallanthus sonchifolius): optimization for fructan retention. LWT - Food Science and Technology, 71, 77-87.

Özkan, G., \& Bilek, S.E (2014). Microencapsulation of natural food colourants. International Journal of Nutrition and Food Sciences, 3 (3),145-156.

Parussolo, G., Busatto, R. T., Schmitt, J., Pauletto, R., Schons, P. F., \& Ries, E. F. (2017). Synbiotic ice cream containing yacon flour and Lactobacillus acidophylus NCFM. LWT-Food Science and Technology, 82,192-198.

Paulo, F; \& Santos, L. (2017). Design of experiments for microencapsulation applications: A review. Materials Science and Engineering: C, 77, $1327-1340$.

Rai, A. K., Kumari, R., S. Sanjukta, S., D., \& Sahoo, S. (2016). Production of bioactive protein hydrolysate using the yeasts isolated from soft chhurpi. Bioresource Technology, 219, 239-245.

Reis, F. R., Marques, C., Moraes, A. C. S. de, \& Masson, M. L. (2021). Effect of processing methods on yacon roots health-promoting compounds and related properties. Trends in Food Science \& Technology, 113, 346-354.

Rezende, Y.R.R.S., Nogueira, J.P., \& Narain, N. (2018). Microencapsulation of extracts of bioactive compounds obtained from acerola (Malpighia emarginata DC) pulp and residue by spray and freeze drying: Chemical, morphological and chemometric characterization. Food chemistry, 254, $281-291$.

Rivera, G. , Bocanegra-garcía, V., \& MONGE, A.(2010). Traditional plants as source of functional foods: a review Plantas tradicionales como fuente de alimentos funcionales: una revisión CyTA-Journal of Food, 8(2), 159-167.

Russo, D., Valentão, P., Andrade, P. B., Fernandez, E. C., \& Milella, L. (2015). Evaluation of antioxidant, antidiabetic and anticholinesterase activities of smallanthus sonchifolius landraces and correlation with their phytochemical profiles. International Journal of Molecular Sciences, $16(8), 17696-17718$.

Santos, S. S., Rodrigues, L. M., Costa, S. C., Bergamasco, R. D. C., \& Madrona, G. S. (2017). Microcapsules of blackberry pomace (Rubus fruticosus): light and temperature stability. Chemical Engineering Transactions. 57, 1837- 1842.

Shi, Q., Zheng, Y., \& Zhao, Y. (2013). Mathematical modeling on thin-layer heat pump drying of yacon (Smallanthus sonchifolius) slices. Energy Conversion and Management, 71, 208-216.

Shishir, M. R. I., \& Chen, W. (2017). Trends of spray drying: a critical review on drying of fruit and vegetable juices. Trends in Food Science \& Technology, 49-67.

Silva,M. A., Albuquerque, T. G., Alves, R. C., Oliveira, M. B. P., \& Costa, H. S. (2020) Melon (Cucumis melo L.) by-products: potential food ingredients for novel functional foods? Trends in Food Science e Technology, 98, 181-189.

Silva, D. M. N, Oliveira, F. L., Cavatte, P. C., Augusto, M., Quaresma, L., \& Christo, B. F. (2018). Growth and development of yacon in different periods of planting and growing regions. Acta Scientiarum. Agronomy, 40, 1-9.

Silva, M. F. G., Dionísio, A. P., Abreu, F. A. P., Pinto, C. O., Silva, L. M. A., Brito, E. S., et al. (2018). Optimization of enzymatic treatment to produce yacon juice clarified by microfiltration with high levels of chlorogenic acid and fructooligosaccharides. Journal of Food Processing and Preservation, 42, 1-8.

Sivieri, K., Morales, M. V., Saad, S. M. I., Adorno, M. A., Sakamoto, I. K., \& Rossi, E. A. (2014) Prebiotic effect of fructooligosaccharide in the simulator of the human intestinal microbial ecosystem (SHIME model). Journal of Medicinal Food, 17, 1-8.

Sosa, N., Gerbino, E., Golowczyc, M. A., Schebor, C., Gómez-zavalia, A., \& Tymczyszyn, E. E. (2016). Effect of Galacto-Oligosaccharides: Maltodextrin Matrices on the Recovery of Lactobacillus plantarum after Spray-Drying. Front Microbioogyl. 7, 584.

Sousa, S., Pinto, J., Pereira, C., Malcata, F. X., Pacheco, M. T. B., Gomes, A. M., \& Pintado, M. (2015). In vitro evaluation of yacon (Smallanthus sonchifolius) tuber flour prebiotic potential. Food and Bioproducts Processing, 95, 96- 105.

Souza, A. L. R., Hidalgo-chávez, D. W., Pontes, S. M, Gomes, F. S, Cabral, L. M. C., \&Tonon, R. V. (2018). Microencapsulation by spray drying of a lycopene-rich tomato concentrate: Characterization and stability. LWT Food Science and Technology, 91, $286-292$.

Tengse, D. D., Priya, B., Kumar, P., \& Arun, R. (2017). Optimization for encapsulation of green tea (Camellia sinensis L.) extract by spray drying technology. Journal of Food Measurement and Characterization, 11, 85-92.

Tontul, I., \& Topuz, A. (2017). Spray-drying of fruit and vegetable juices: Effect of drying conditions on the product yield and physical properties. Trends in Food Science \& Technology, 63, 91-102.

Vila, M. M., Chaud, M. V., \& Balcao, V. M. (2015). Microencapsulation of natural anti-oxidant pigments. In L. M. C. Sagis (Ed.), Microencapsulation and microspheres for food applications, 369-389.

WHO. World Health Organization. 2019. Overweight and obesity https://www.who.int/emergencies/diseases/novelcoro navirus2019?gcli d=CjwKCAiAudD_BRBXEiwAudakX3myRIRIJcetUh4AxWiN8Y qYjJZ61cVygqKSnGpzQ9zY-SAlmp9L-hoCpf4QAvD_BwE>>

Yasmin, A., Butt, M. S., Afzaal, M., Baak, M., Nadeem, M. T., \& Shahid, M. Z. (2015). Prebiotics, gut microbiota and metabolic risks: Unveiling the relationship.Journal of Functional Foods, 17, 189-201.

Zardini, E. (1991) Ethnobotanical notes on“Yacon”Polymnia sonchifolia (Asteraceae). Economic Botany, 45 (1),72-85.

Zhen-yuan, Z. H. U., Jing-yi, Z., Li-jing, C., Xiao-cui, L. I. U., Yang, L. I. U., Wan-xiao, W., \&Yong-min, Z. (2014) Comparative evaluation of polysaccharides isolated from Astragalus, oyster mushroom, and yacon as inhibitors of $\alpha$-glucosidase. Chinese Journal of Natural Medicines,12 (4), $290-293$. 